\title{
Analysis of Two Stage Folded Cascode Operational Amplifier in 90nm Technology
}

\author{
Jasbir Kaur ${ }^{1}$, Neha Shukla ${ }^{2}$ \\ Assistant Professor, P.E.C University of Technology, Chandigarh, India ${ }^{1}$ \\ P.G Scholar, P.E.C University of Technology, Chandigarh, India ${ }^{2}$
}

\begin{abstract}
Folded cascode configuration is a very fascinating unit to any Analog Vlsi researcher as it has advantage of high gain, lesser power dissipation and High Bandwidth. This paper describes about the parametric analysis of folded cascode opamp at low power supply requirements, input voltage and using $90 \mathrm{~nm}$ technology. At the end, the results which are obtained are compared with the conventional two stage opamp with the same specifications and same technology (90nm).
\end{abstract}

Keywords: CMRR, dB, NMOS, PMOS, opamp, folded cascade.

\section{INTRODUCTION}

Operational amplifiers are playing prominent role in many electronics applications. These amplifiers are important as these are very high gain amplifiers. Basically, an ideal opamp characteristics becomes the driving force behind more and more improvements in practical opamps. Folded cascode is one of the effort in the field of enhancing the performance of practical opamp so that the characteristics of ideal opmap can be achieved.

This paper is divided into four sections. First section describes about basics of conventional two stage opamp in detail. The second section describes about Folded Cascode configuration along with difference between normal cascode and folded cascade. The third gives the detailed observations and results which are obtained from simulations in cadence. The results are then compared with the conventional opamp with various parameters. Finally in last section this paper gives the conclusion

\section{BASIC APPROACH TOWARDS FOLDED CASCODE OPERATIONAL AMPLIFIER}

A. The Conventional Two Stage Operational Amplifier

Basic two stage opamp is basically combination of two stages

1. Differntial stage

2. High gain stage

In nutshell, it can be said that the two stage opmap is a high gain direct coupled amplifier. Verstality of opamp is observed when lots of applications are encountered like oscillators, filters, also in regulated power supply. The only requirement is that accurate type of feedback has to be selected

1. Positive feedback for Oscillators

2. Neagative Feedback for Amplification Following figure 1 is showing the two stage opamp.

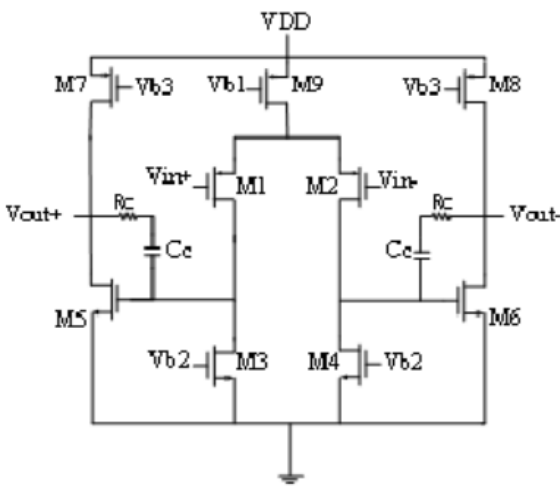

Figure 1: Two Stage CMOS Operational Amplifier 
B. Cascode Configurations

There are two types of cascode configurations:

1. Telescopic

2. Folded Cascode

Telescopic cascode configuration is very useful for neural recording applications .In this MOS transistors are stacked such that gain is improved and to increase overall output impedance .Practically, NMOS transistors are usually preferred over PMOS so as to achieve high unity gain bandwidth and high transconductance and these can be obtained by using NMOS transistors. But if noise suppression and improved phase margin is required then PMOS is the best option. Telescopic amplifier is best described by figure 2 .

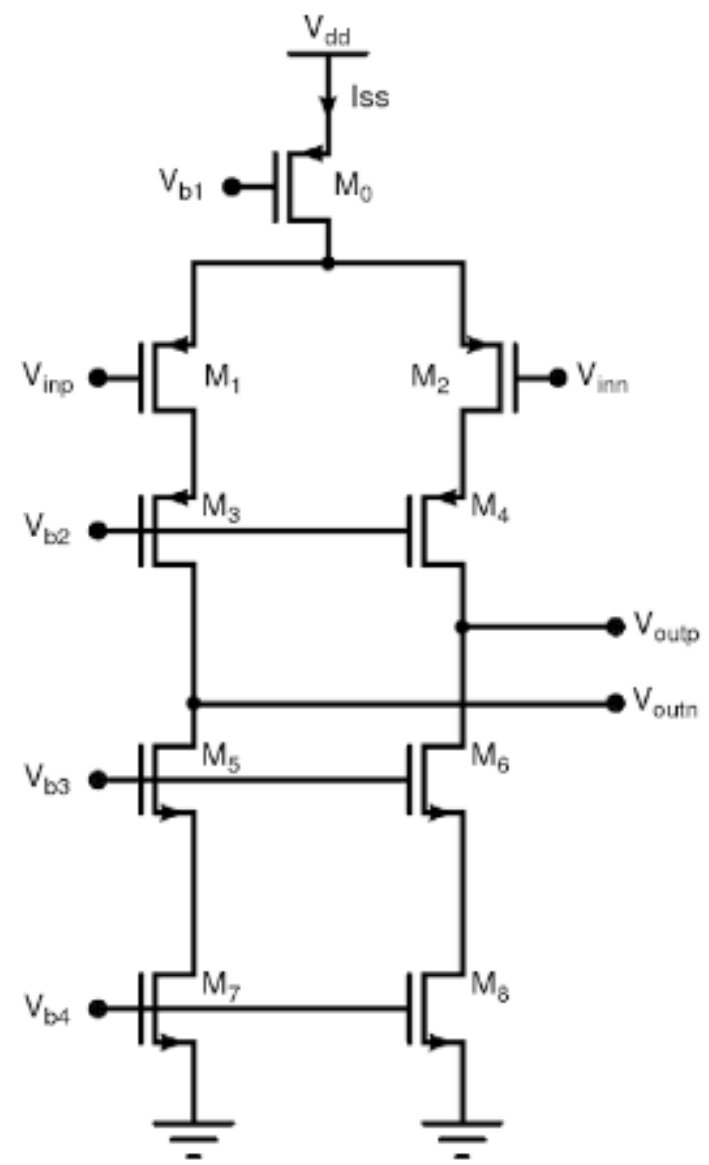

Figure 2: Telescopic CMOS Operational Amplifier

C. Introduction to Folded Cascode Configuration

Before analyzing the folded cascode, there is a need to see the term 'cascode' means. The Cascode Stage is nothing but the combination of Common Source and Common Gate Stage.

These Kind of configurations is used to achieve:

1. Boosted Gain opamp

2. High Transconductance, As this stage increases the output imapedance to a great extent .The Cascode Configuration can be studided in two parts

1. Normal Cascode

2. Folded Cascode

Normal Cascode: Normal cascode is a combination of CS-CG stage in series configuration. The main requirement is the both the transistors must be same either $\mathrm{p}$ channel or $\mathrm{n}$ channel It is shown in figure. Advantages of Normal Cascode The biggest advantage of normal cascode is that it can increase gain 2-3 folds depends upone the number of stages which are cascoded. The second big advantage of folded cascode opamp is these are very good with layouts.

In refernce to fig 3 , there is only one pair, it can be extended to $\mathrm{n}$ times depending upone the need of gain which is required. 


\section{International Journal of Innovative Research in} Electrical, Electronics, Instrumentation and Control Engineering

\section{ISO 3297:2007 Certified}

Vol. 5, Issue 6, June 2017

The various disadvantages of Normal Cascode are

1. The power supply requirement is very high

2. The GBW product is not constant, as the gain increases with this configuration, the bandwidth decreases as a result of which the GBW is not constant.

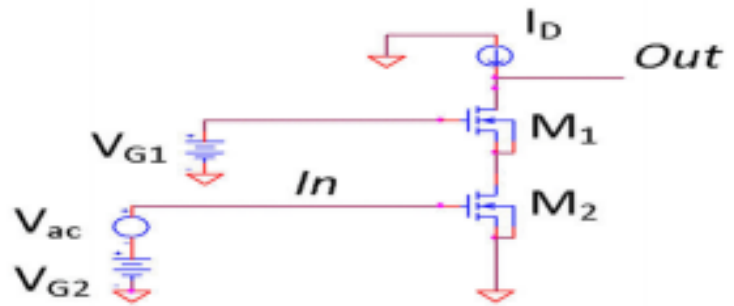

Figure 3 : Normal Cascode Configuration

Folded cascode configuration is an extention of normal cascode configuration. The main difference is that this configuration consists of two transistors either $\mathrm{n}$ channel and $\mathrm{p}$ channel or both $\mathrm{n}$ channel transistors in a way that both transistors must face each other.It will be more clear from figure 4 that this folded cascode as it is clear from figure 4 that both the transistors MP1 MP2 are placed opposite to each other. Similarly it is extended one more time with MP3 MP4.

Advantages of Folded Cascode

1. The biggest advantage of folded cascode is that the power supply requirement is low.

2. The GBW is constant upto an extent.

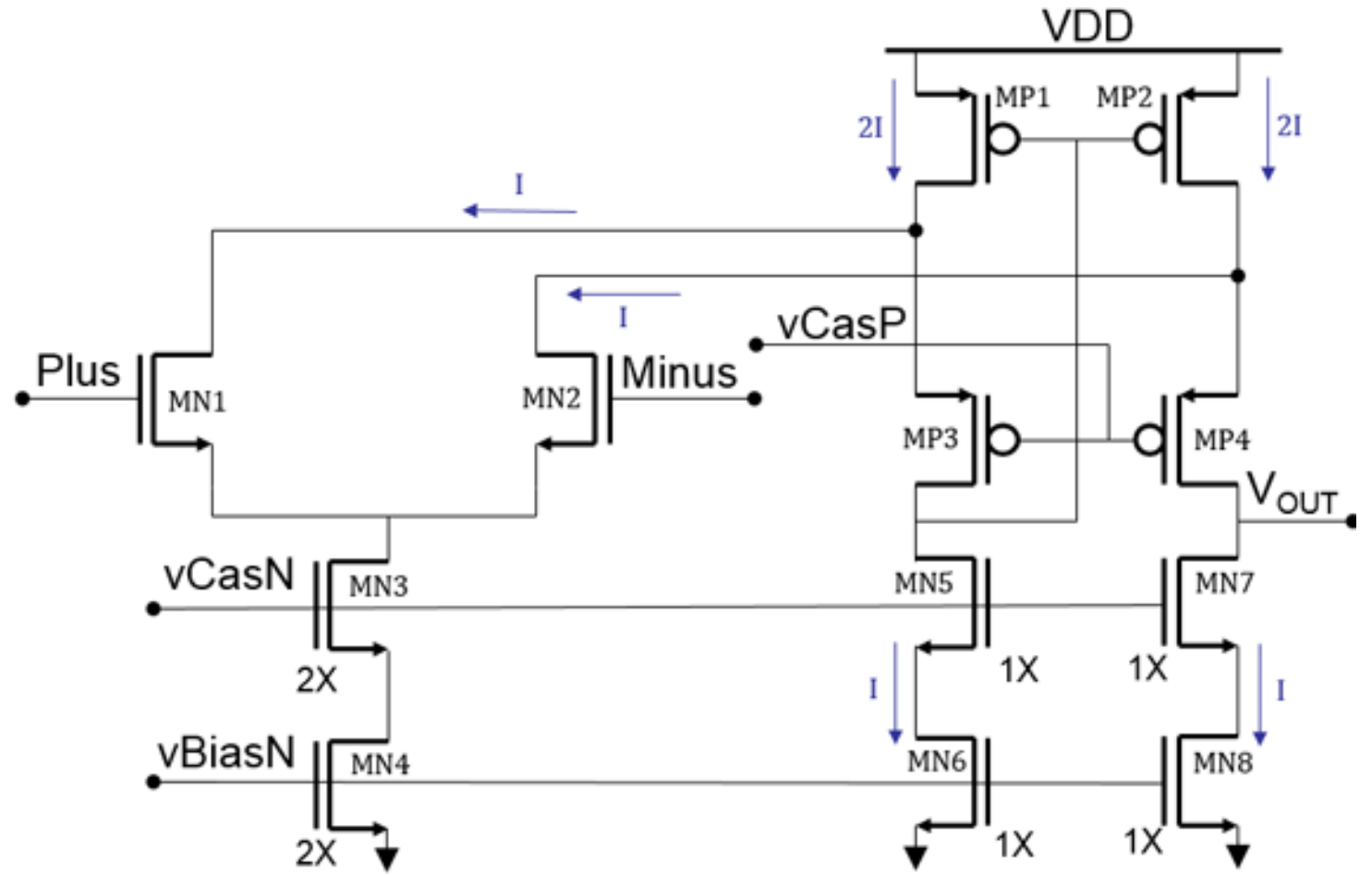

Figure 4 : Folded Cascode Configuration.

D. Design Methodology for Two stage Opamp Folded Cascode opamp

1) Selection of W/L Ratio

Out of all the factors W/L ratio is one of the most important parameter in designing any analog device. So in $90 \mathrm{~nm}$ technology, W/L for NMOS for PMOS is 1.2.

2) Selection of Power Supply

The power supply is $1.8 \mathrm{~V}$

3) Selection of Input Signal

The input signal should be given between 1 to $1.2 \mathrm{~V}$ 
IJIREEICE

International Journal of Innovative Research in Electrical, Electronics, Instrumentation and Control Engineering

ISO 3297:2007 Certified

Vol. 5, Issue 6, June 2017

\section{III.VARIOUS ANALYSIS OF FOLDED CASCODE TWO STAGE OPAMP}

1) Bandwidth: The gain and frequency are inveresly proportional to each other. The bandwidth is observed by using AC analysis . It also defines the speed of the circuit.The BW of folded cascode is $96.94 \mathrm{MHz}$.

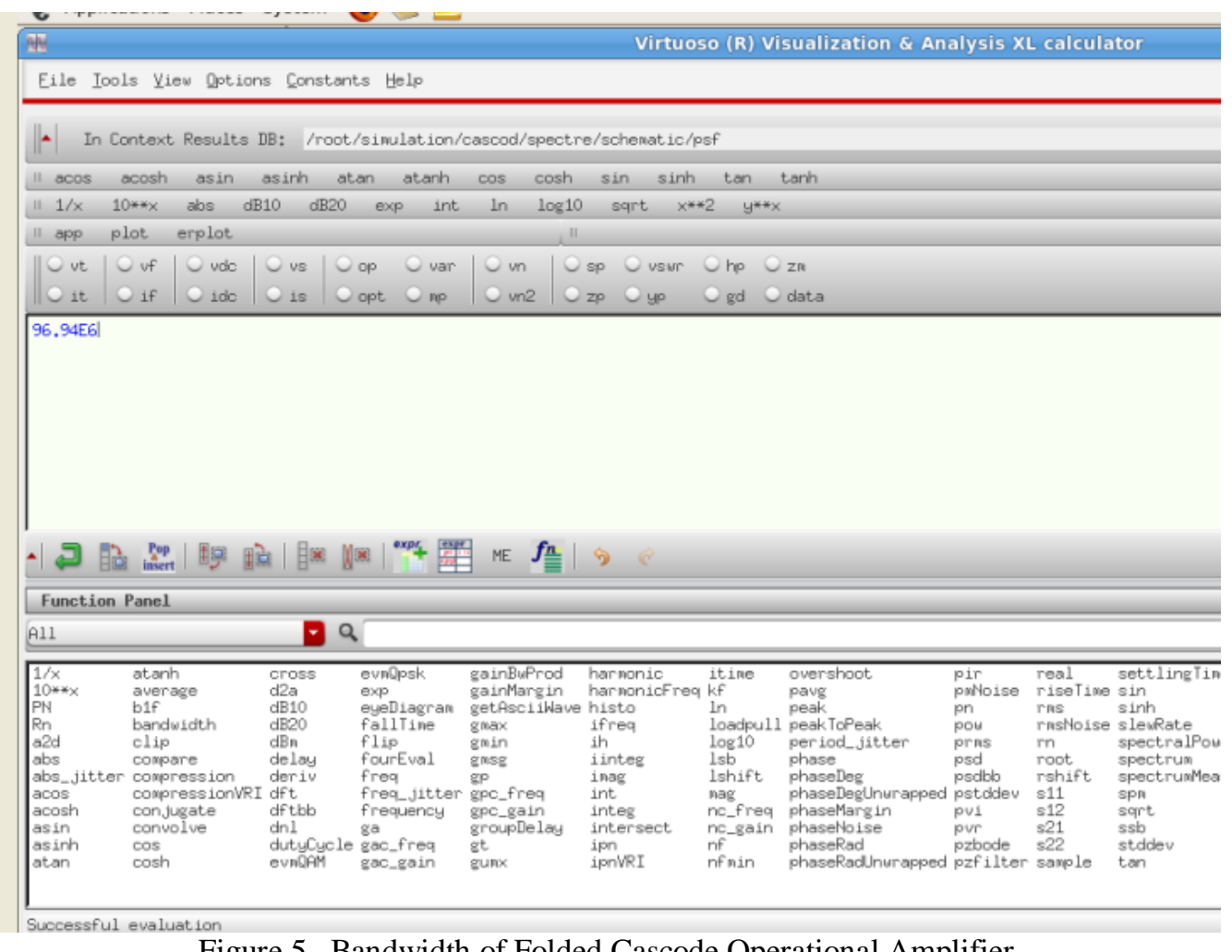

Figure 5 . Bandwidth of Folded Cascode Operational Amplifier

2) Phase Margin: As it is already discussed that folded cascode configuration gives very high gain and If the phase margin of opamp is grater than 60(in degrees), it is acceptable for analog applications. This is analysed using AC analysis Methodology.

It is shown in figure 6 : The observed gain is $80 \mathrm{~dB}$ and phase margin $=71$ (in degrees).

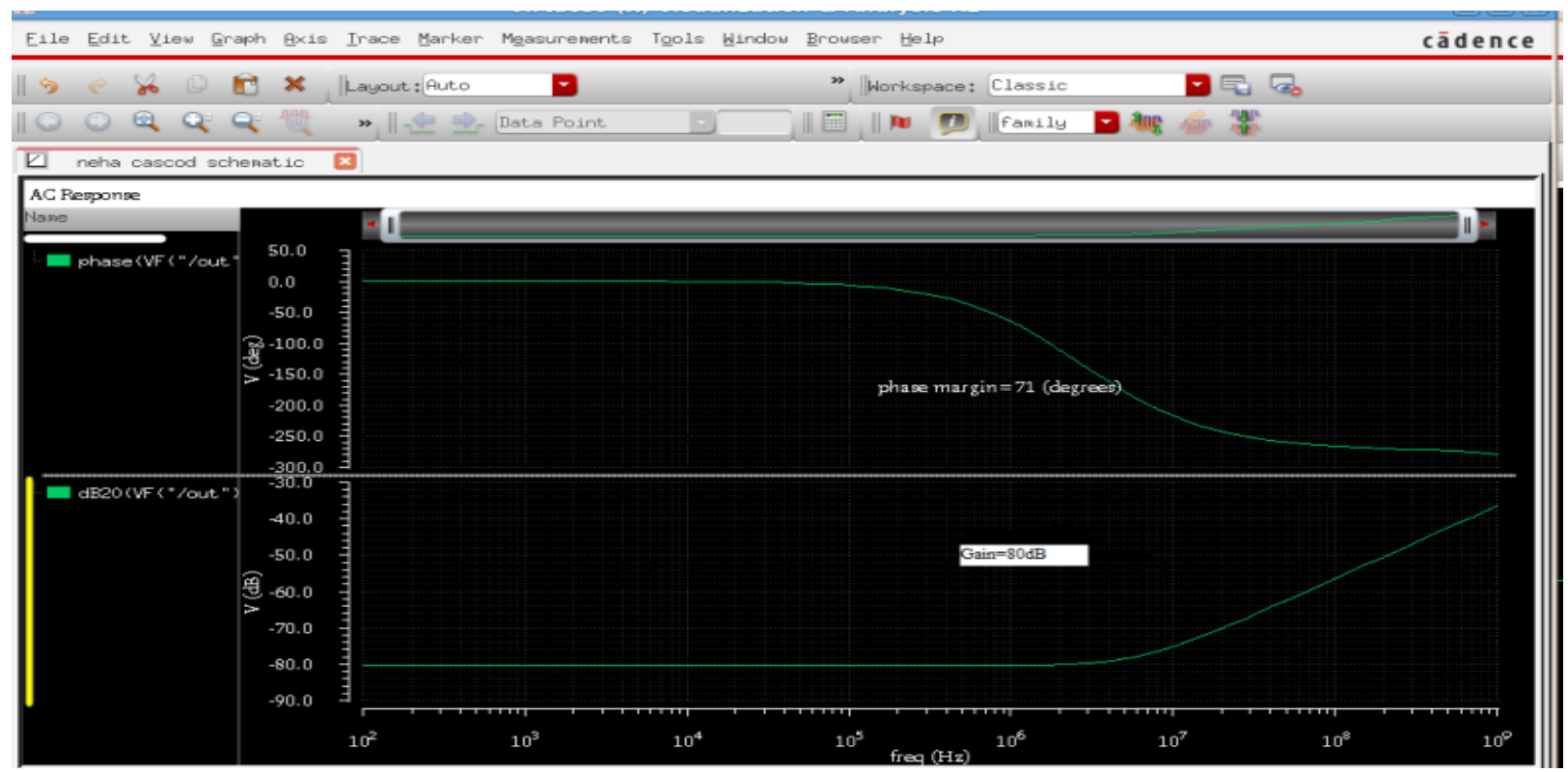

Figure 6: Gain \& Phase of Folded Cascode Configuration. 


\section{International Journal of Innovative Research in} Electrical, Electronics, Instrumentation and Control Engineering

ISO 3297:2007 Certified

Vol. 5, Issue 6, June 2017

3) Slew Rate: The slew rate is basically defined as the the rate of change of output voltage with respect to time. It is analyzed by using transient analysis. The slew rate of folded cascode is shown in fig 7.

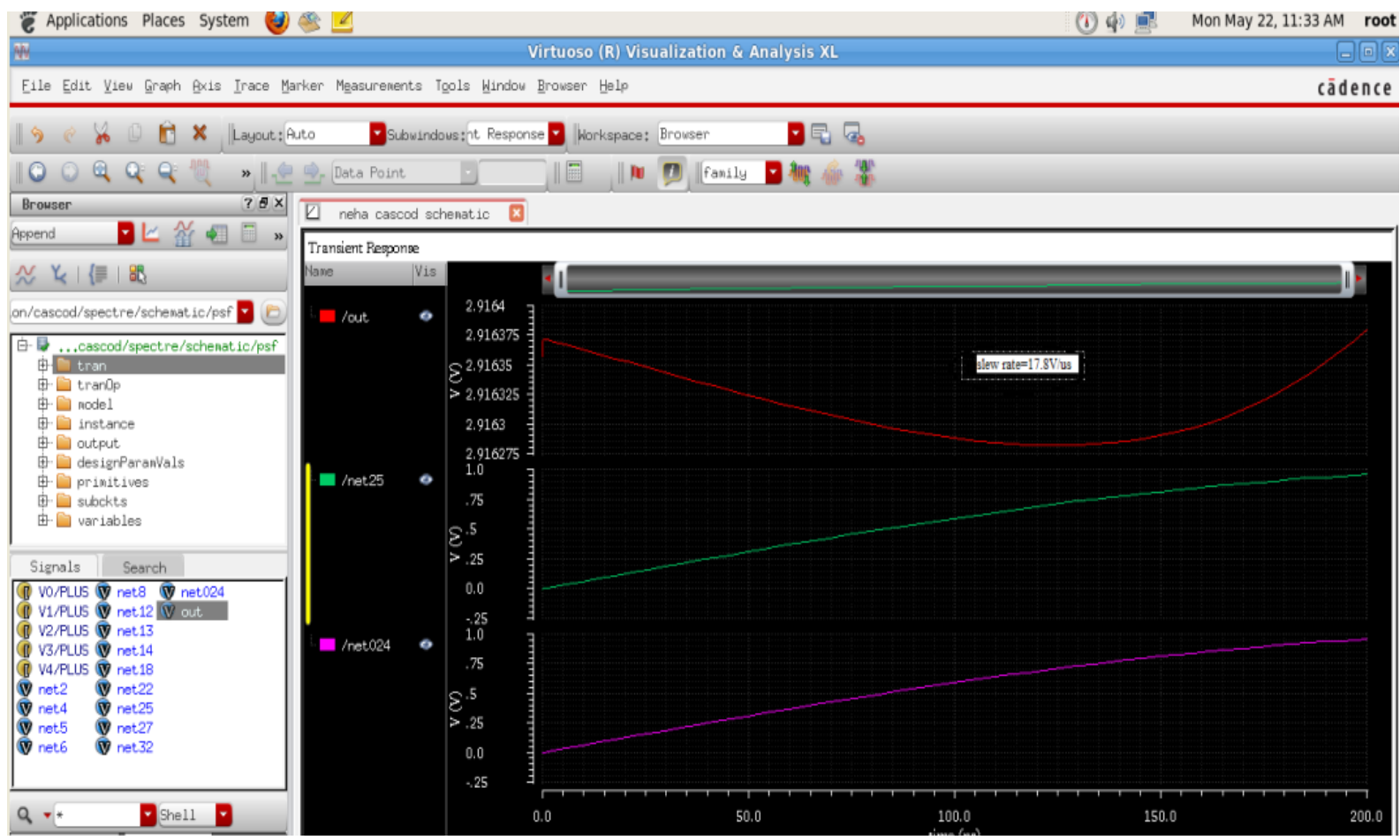

Figure 7: Slew Rate of Folded Cascode Configuartion

The slew rate of two stage opamp in 90nm technology is shown in figure 8

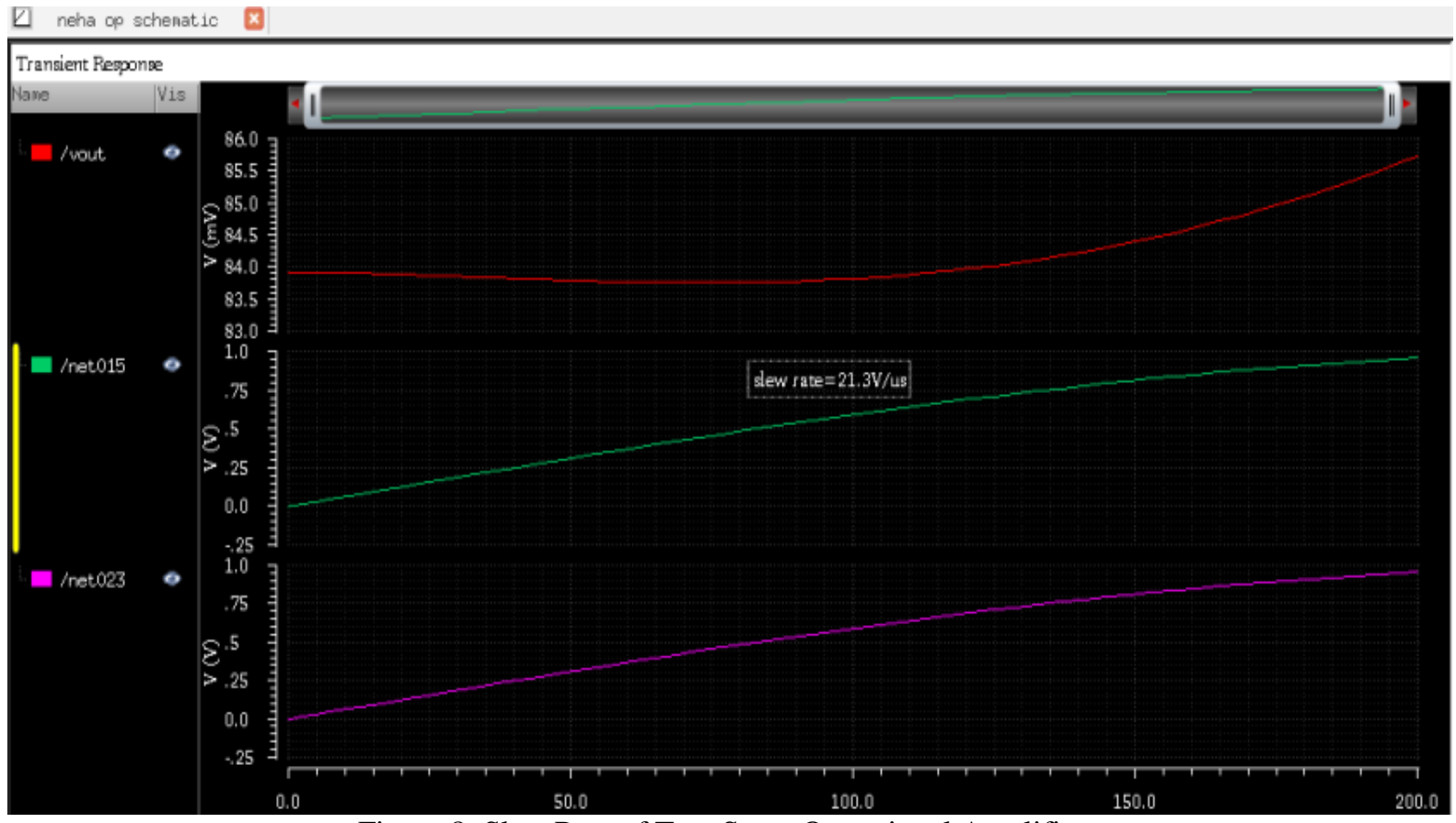

Figure 8: Slew Rate of Two Stage Operational Amplifiers

4) Power Dissipation: The power dissipation should be as low as possible for a good circuit. The power dissipation of folded cascode is shown below in figure 9 the power dissipation of conventional two stage opamp is shown in figure 10 . 


\section{International Journal of Innovative Research in} Electrical, Electronics, Instrumentation and Control Engineering

ISO 3297:2007 Certified

Vol. 5, Issue 6, June 2017

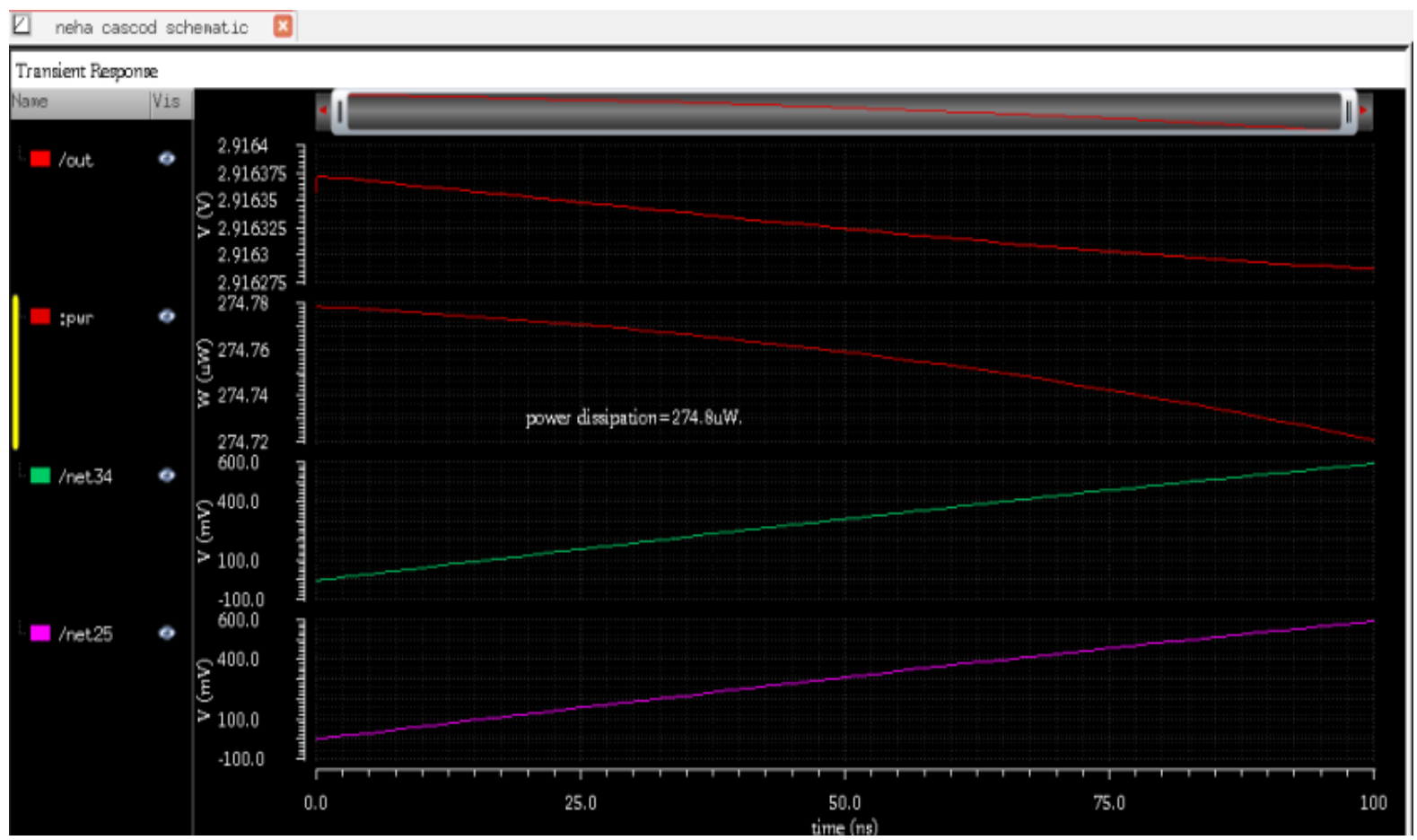

Figure 9: Power Dissipation of Folded Cascode Operational Amplifier.

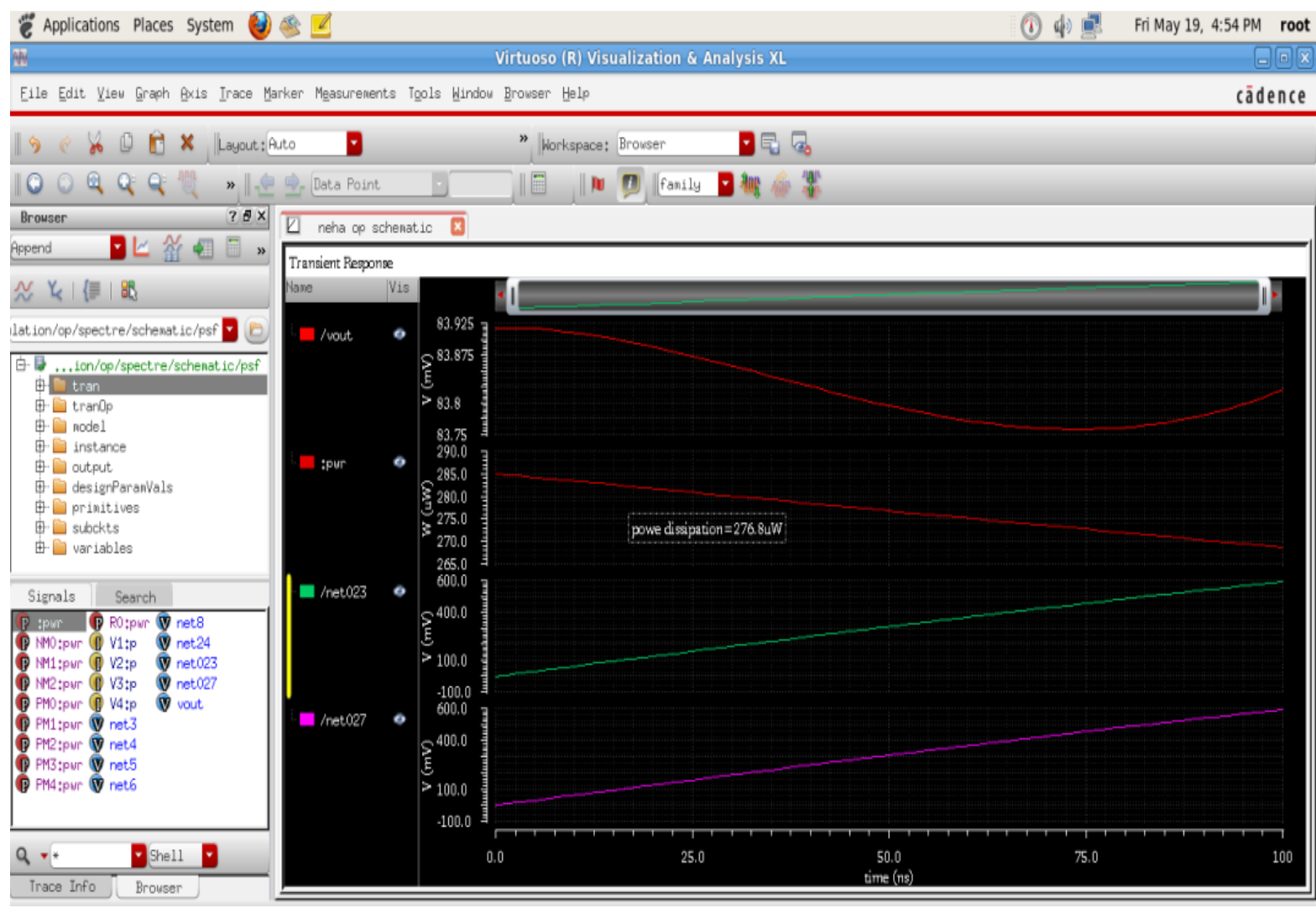

Figure 10 : Power Dissipation of Two Stage Operational Amplifier.

E. DC Analysis of Folded Cascode opamp

With DC analysis, any operating point of transistors involved in the circuit cab be observed and these operating parameters can be varied as per the requirement. DC analysis is shown in figure 11 


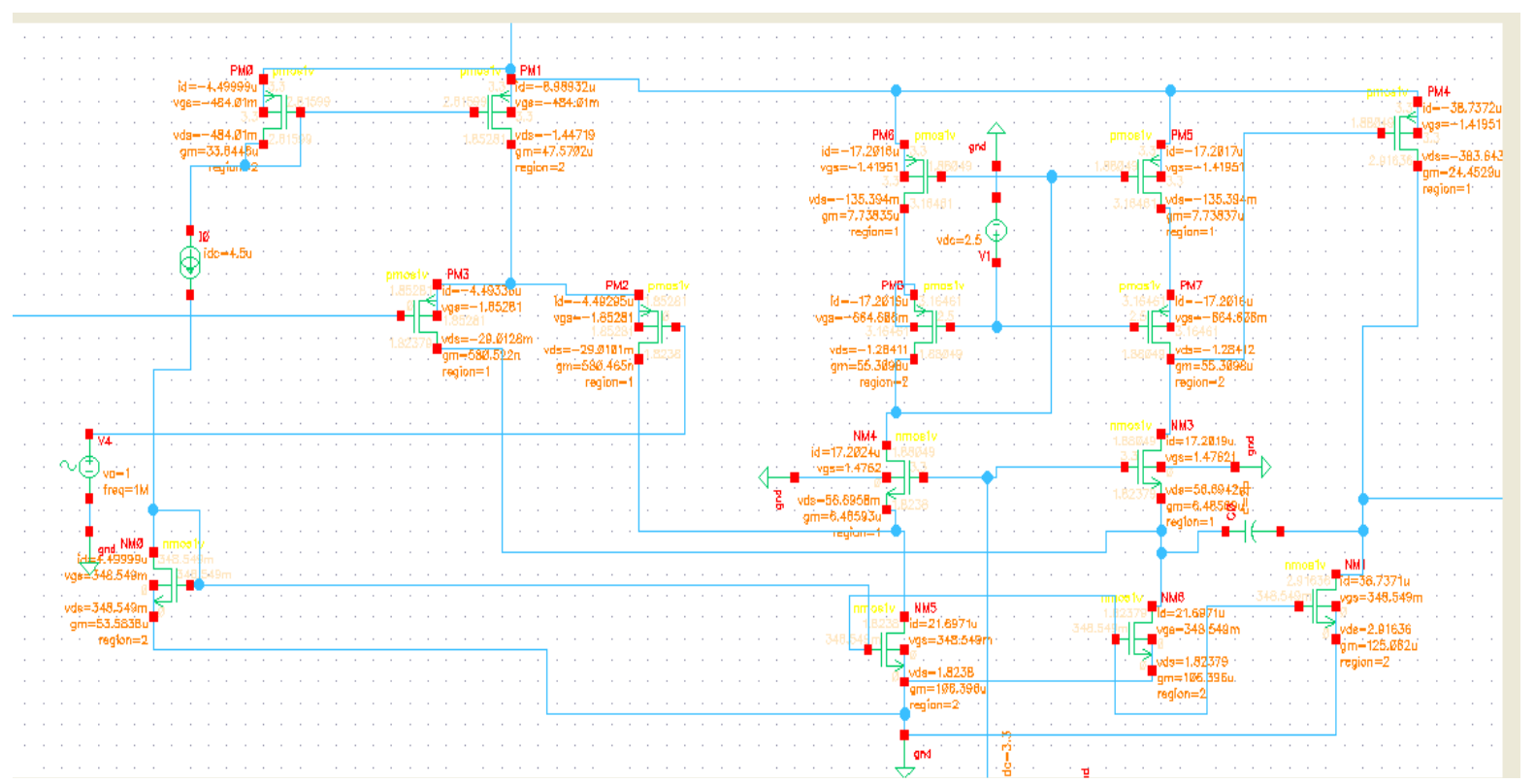

Figure 11: DC analysis of Folded Cascode Operational Amplifier

TABLE I COMPARISION BETWEEN VARIOUS PARAMETERS

\begin{tabular}{|l|l|l|l|l|}
\hline \multirow{2}{*}{$\begin{array}{l}\text { S. } \\
\text { No }\end{array}$} & After A.C \& D.C Analysis & Reference Paper \\
\cline { 2 - 5 } & Parameters & $\begin{array}{l}\text { Conventional } \\
\text { Opamp }\end{array}$ & $\begin{array}{l}\text { Folded } \\
\text { Cascode } \\
\text { Opamp }\end{array}$ & $\begin{array}{l}\text { Design of Folded Cascode } \\
\text { Operational Amplifier in High } \\
\text { Voltage CMOS Technology [10] }\end{array}$ \\
\hline 1 & Power Supply(V) & 1.8 & 1.8 & 20 \\
\hline 2 & Power Dissipation $(\mu \mathrm{W})$ & 276.8 & 274.8 & 0.02212 \\
\hline 3 & Phase Margin(in degrees) & 125 & 71 & 52.09 \\
\hline 4 & Bandwidth(MHz) & 0.09042 & 96.94 & 17.80 \\
\hline 5 & Capacitance $(\mathrm{pF})$ & 30 & 2 & 10 \\
\hline 6 & Gain $(\mathrm{dB})$ & 22.14 & 80 & 84.52 \\
\hline 7 & Slew Rate9(V/ $\mu$ s) & 21.3 & 17.8 & 25.69 \\
\hline 8 & CMRR(dB) & Approx80 & 160 & 97.95 \\
\hline 9 & Input signal Voltage(V) & 1 & 1 & - \\
\hline 10 & Frequency of input signal $(\mathrm{MHz})$ & 1 & 1 & - \\
\hline
\end{tabular}

\section{IV.CONCLUSION}

The results obtained for folded cascode opamp and conventional opam in 90nm are:

1. Power dissipation is improved from $276.8 \mathrm{uW}$ to $274.8 \mathrm{uW}$

2. Gain is improved from $22 \mathrm{~dB}$ to $80 \mathrm{~dB}$

3. Slew rate is also improved $21.3 \mathrm{~V} / \mathrm{us}$ to $17.8 \mathrm{~V} / \mathrm{us}$.

4. CMRR is also improved from $80 \mathrm{~dB}$ to $160 \mathrm{~dB}$.

Hence, Folded Cascode opamp can be used where the higher gain is required

\section{ACKNOWLEDGMENT}

Working on this research paper was a source of immense knowledge to us. We would like to thank God, our parents for their love and support.

\section{REFERENCES}

[1] J. Mahattanaku, Design Procedure for Two-Stage CMOS OperationalAmplifiers Employing Current Buffer, IEEE Transactions on Circuits and SystemsII: Express Briefs, Vol. 52,pp.766-770, November 2005

[2] Ayush Gupta, Aditya Bhansali, Swati Bhargava, Shruti Jain Configuration of Operational Amplifier using CMOS 


\section{IJIREEICE

[3] S. Zhang, C. Zhu, J. K. O. Sin, and P. K. T. Mok, “A novel ultrathin elevated channel low-temperature poly-Si TFT,” IEEE Electron Device Lett., vol. 20, pp. 569-571, Nov. 1999.

[4] D. Nageshwarrao, K.Suresh Kumar, Y.Rajasree Rao,G.Jyothi, Implementation and simulation of CMOS two stage operational amplifier, International Journal of Advances in Engineering Technology, Vol. 5,pp.162-167, Jan. 2013

[5] A.-J. Annema, B. Nauta, R. van Langevelde, and H. Tuinhout, Analog circuits in ultra-deep-submicron cmos, IEEE J. Solid-State Circuits, vol.Vol. 40, January 2005

[6] E. Sackinger and W. Guggenbuhl, A high-swing high-impedance mos cascode circuit, IEEE J. Solid-State Circuits, vol. 25, pp. 289298, Feb.1990

[7] J. Lloyd and H.-S. Lee, A cmos op amp with fully-differential gainenhancement,IEEE, 1994

[8] T. Burger and Q. Huang, On the optimum design of regulated cascode operational transconductance amplifiers, ISLPED, 1998.

[9] NPTEL lecture on Analog Vlsi design (IIT Bombay)by Prof. A.N Chandorkar

[10] T. Burger and Q. Huang, A $100 \mathrm{db}, 480 \mathrm{mhz}$ ota in $0.7 \mathrm{~m} \mathrm{cmos}$ for sampled-data applications, Custom Integrated Circuits Conference, 1996.

[11] Benjamin Lutgen, 2009," Design of a folded cascode operational amplifier in high voltage CMOS technology".

\section{BIOGRAPHIES}

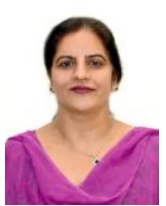

JASBIR KAUR She is an Assistant Professor at P.E.C University of Technology, Chandigarh. She has more than 20 publications. She is pursuing her PhD in VLSI Design at P.E.C University of Technology.

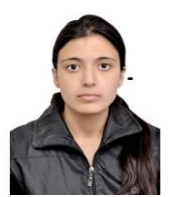

NEHA SHUKLA She is P.G Scholar at P.E.C University of Technology Chandigarh. She has also published her paper in ICAMT-2016 (Elsevier indexed) \& ICMTES-2017 (Scopus Indexed). 